\title{
Exercise stress echocardiography of the pulmonary circulation and right ventricular- arterial coupling in healthy adolescents
}

\author{
Kevin Forton (1) ${ }^{1,2, *}$, Yoshiki Motoji ${ }^{1,2}$, Sergio Caravita ${ }^{3,4}$, Vitalie Faoro ${ }^{1}$, and \\ Robert Naeije ${ }^{1}$
}

\begin{abstract}
${ }^{1}$ Faculty of Motor Sciences, Cardio-Pulmonary Exercise Laboratory, Université Libre de Bruxelles, Erasmus Campus CP 604, 808 Lennik Road, 1070 Brussels, Belgium; ${ }^{2}$ Department of Cardiology, Erasmus University Hospital, 1070 Brussels, Belgium; ${ }^{3}$ Department of Cardiovascular, Neural and Metabolic Sciences, Istituto Auxologico Italiano IRCCS Ospedale San Luca, Piazzale Brescia 20, 20149 Milano, Italy; and ${ }^{4}$ Department of Management, Information and Production Engineering, University of Bergamo, Viale G. Marconi, 5, 24044 Dalmine (BG), Italy
\end{abstract}

Received 3 February 2020; editorial decision 1 April 2020; accepted 4 April 2020; online publish-ahead-of-print 13 April 2020

Aims

To explore the effects of age and sex in adolescents vs. young or middle-aged adults on pulmonary vascular function and right ventricular-arterial (RV-PA) coupling as assessed by exercise stress echocardiography.

Methods and results

Forty healthy adolescents aged 12-15 years were compared with 40 young adults aged 17-22 years and 40 middleaged adults aged 30-50years. Sex distribution was equal in the three groups. All the subjects underwent an exercise stress echocardiography. A pulmonary vascular distensibility coefficient $\alpha$ was determined from multipoint pulmonary vascular pressure-flow relationships. RV-PA coupling was assessed by the tricuspid annular plane systolic excursion (TAPSE) to systolic pulmonary artery pressure (PASP) ratio, who has been previously validated by invasive study. While cardiac index and mean PAP were not different, adolescents compared to young and middle-aged adults, respectively had higher pulmonary vascular distensibility coefficients $\alpha(1.60 \pm 0.31 \% / \mathrm{mmHg}$ vs. $1.39 \pm 0.29 \% /$ $\mathrm{mmHg}$ vs. $1.20 \pm 0.35 \% / \mathrm{mmHg}, P<0.00001)$. Adolescents and young adults compared to middle-aged adults, respectively had higher TAPSE/PASP ratios at rest $(1.24 \pm 0.18 \mathrm{~mm} / \mathrm{mmHg}$ and $1.22 \pm 0.17 \mathrm{~mm} / \mathrm{mmHg}$ vs. $1.07 \pm 0.18 \mathrm{~mm} / \mathrm{mmHg}, P<0.008)$ and during exercise $(0.86 \pm 0.24,0.80 \pm 0.15$ and $0.72 \pm 0.15 \mathrm{~mm} / \mathrm{mmHg}, P<0.04)$.

The TAPSE/PASP ratio decreased with exercise. There were no sex differences in $\alpha$ or TAPSE/PASP.

Conclusion Compared to adults, adolescents present with a sex-independent more distensible pulmonary circulation. Resting and exercise RV-PA coupling is decreased in middle-aged adults.

Keywords

pulmonary vascular resistance $\bullet$ growth $\bullet$ children $\bullet \mathrm{VO}_{2} \mathrm{max} \cdot \mathrm{TAPSE}$

\section{Introduction}

Pulmonary vascular pressure-flow relationships are slightly curvilinear, rather than strictly linear as assumed by the pulmonary vascular resistance (PVR) equation, and this is explained by the natural distensibility of pulmonary resistive vessels. ${ }^{1}$ Accordingly, the PVR equation can be improved by the incorporation of a resistive vessel distensibility coefficient $\alpha$ so that PAMP $=\left[\left((1+\alpha \mathrm{LAP})^{5}+5 \alpha \cdot \mathrm{R}_{0} \cdot \mathrm{CO}\right)^{1 / 5}-1\right] /$ $\alpha$, where PAMP is pulmonary artery mean pressure, LAP left atrial pressure, CO cardiac output, and $\mathrm{R}_{0}$ PAMP/CO at rest. ${ }^{2}$ An interesting application of this equation is that $\alpha$ can be calculated from the best fit of PAMP, LAP (or wedged PAP, PAWP), and $\mathrm{CO}$ measurements obtained during exercise to increase cardiac output. ${ }^{2}$ Invasive and non-invasive studies have shown that a so calculated index of distensibility $\alpha$, is normally between 1 and $2 \% / \mathrm{mmHg}$, higher in young healthy women, and decreased with aging or chronic hypoxic exposure., ${ }^{3,4}$ Exercise haemodynamic studies have shown that the distensibility coefficient $\alpha$ is decreased in early or latent pulmonary vascular disease ${ }^{5}$ and in heart failure. ${ }^{6}$ In the latter study, the $\alpha$ coefficient was positively correlated to right ventricular ejection fraction (RVEF) and independently predicted peak oxygen uptake $\left(\mathrm{VO}_{2}\right)$ suggesting $\mathrm{RV}$ afterload limitation of maximum 
CO. ${ }^{6}$ There has been no report of $\alpha$ coefficient determinations in children.

The coupling of RV function to the pulmonary circulation is best defined by a ratio of end-systolic to arterial elastances (Ees/Ea). ${ }^{7,8}$ Gold-standard measurements of Ees/Ea require sophisticated highfidelity micromanometer-tipped conductance catheter technology to generate pressure-volume relationships. This is not practical for bedside and even less so for exercise studies. Therefore, echocardiographic surrogates have been developed.' It has been recently suggested that the best echocardiographic estimate of RV Ees/Ea is the tricuspid annular plane systolic excursion (TAPSE) to systolic PAP (PASP) ratio. ${ }^{10}$ The TAPSE/PASP is a potent predictor of outcome in heart failure and in pulmonary hypertension. ${ }^{11}$ The TAPSE/ PASP decreases with aging and with exercise. ${ }^{12,13}$ However, there has been no report of TAPSE/PASP ratio measurements in children.

In this study, we evaluated the RV and the pulmonary circulation during exercise in adolescent children as compared to young and middle-aged adults.

\section{Methods}

\section{Study population}

The study included 120 healthy Caucasian subjects from 12 to 50 years old, divided into three age-groups: 12-15, 17-22, and 30-50years old (Table 1). Sex distribution was equal in the three groups. All the subjects were Caucasians, naturally conceived, non-smoker, and declared themselves in good health. They led a healthy lifestyle, with $2-4 \mathrm{~h}$ physical activity per week. None of them was engaged in competitive sports. Their physical examination and resting electrocardiogram were normal. Twenty-one subjects with insufficient quality resting echocardiography had been excluded in the screening phase of the study. The subjects (and parents of the adolescents) gave an informed consent to the study, which was approved by the local Institutional Ethics Committee (reference B406201422389).

\section{Experimental protocol}

Each subject reported to the laboratory on two occasions within a week. On the first visit, the participant underwent a clinical examination and a standard resting echocardiographic examination followed by an incremental stress echocardiography. The exercise echocardiography was performed on a semi-recumbent position as previously reported ${ }^{14}$ with an incremental workload increase of $20 \mathrm{~W} / 2 \mathrm{~min}$ until exhaustion. The

\section{Table I Baseline characteristics of adolescents and young and middle-aged adults}

\begin{tabular}{|c|c|c|c|}
\hline & Adolescents & Young adults & Middle-age adults \\
\hline N & 40 & 40 & 40 \\
\hline Age (years) & $13 \pm 1$ & $18 \pm 2^{*}$ & $39 \pm 8^{* * * *}$ \\
\hline Height (cm) & $164 \pm 9$ & $171 \pm 9 *$ & $173 \pm 9 *$ \\
\hline Weight (kg) & $52 \pm 8$ & $64 \pm 9 *$ & $67 \pm 10^{*}$ \\
\hline $\mathrm{BMI}\left(\mathrm{kg} / \mathrm{m}^{2}\right)$ & $19.3 \pm 3.0$ & $21.9 \pm 2.6^{*}$ & $22.3 \pm 2.0 *$ \\
\hline $\operatorname{BSA}\left(m^{2}\right)$ & $1.85 \pm 0.18$ & $2.09 \pm 0.19 *$ & $2.14 \pm 0.21 *$ \\
\hline
\end{tabular}

BMI, body mass index; BSA, body surface area. $* P<0.001$ vs. adolescents.

$* * P<0.001$ middle-age adults vs. young adults. second visit consisted of a standard incremental cardiopulmonary exercise test (CPET) on a cycle ergometer to measure ventilation $\left(V_{E}\right)$, carbon dioxide output $\left(\mathrm{VCO}_{2}\right)$, and oxygen uptake $\left(\mathrm{VO}_{2}\right)$. Maximum $\mathrm{VO}_{2}$ $\left(\mathrm{VO}_{2} \mathrm{max}\right)$ was considered to be achieved when two of the following criteria were met: an increase in $\mathrm{VO}_{2}$ of $<100 \mathrm{~mL} / \mathrm{min}$ with a further increase in workload, a respiratory exchange ratio (RER) greater than 1.1 or achievement of age-predicted maximal heart rate (HR).

\section{Clinical assessment}

The clinical assessment included a medical history with a clinical examination including measurements of weight (standing weighting scale) and height (wall-mounted stadiometer), blood pressure (BP) (sphygmomanometry), pulse oximetry $\mathrm{O}_{2}$ saturation $\left(\mathrm{SpO}_{2}\right)$ (Nellcor Puritan Bennett Inc., Pleasanton, CA, USA), and electrocardiogram.

\section{Echocardiography}

Echocardiography was performed with a portable echocardiography system (CX50 CompactXtreme Ultrasound System; Philips, Amsterdam, The Netherlands) on an exercise table (Ergoselect II 1200; Ergoline; Bitz, Germany) tilted $35^{\circ}$ backwards and $33^{\circ}$ to the left as used for optimal sampling of signals, as previously reported. ${ }^{15}$ The following measurements and derived calculations were collected at rest and during the second minute at every workload: peak systolic RV-right atrium (RA) pressure gradient, calculated according to the simplified Bernoulli equation $\left(4 \mathrm{~V}^{2} ; \mathrm{V}=\right.$ peak systolic velocity of tricuspid regurgitation flow in continuous wave Doppler); PASP, measured by adding to RV-RA gradient an estimation of RA pressure according to diameter and collapsibility of the inferior vena cava; PAMP estimated as $0.61 \times$ PASP $+2 \mathrm{mmHg},{ }^{16}$ LAP estimated from the ratio of Doppler mitral $E$ flow-velocity wave and tissue Doppler mitral annulus flow (e') early diastolic peak velocity (LAP= $\left.1.9+1.24 \mathrm{E} / \mathrm{e}^{\prime}\right)$; stroke volume (SV) estimated from left ventricle outflow tract (LVOT) area and velocity-time integral (VTI): SV $=(3.14 / 4 \times$ LVOT diameter $\left.^{2}\right) \times \mathrm{VTI}$; CO calculated as SV $\times \mathrm{HR}$ and cardiac index $(\mathrm{Cl})$ by CO/body surface area (BSA); TAPSE recorded by M-mode echocardiography; pulsed tissue Doppler tricuspid annular velocity $S$ ' acquired from the lateral tricuspid annulus in the apical four-chamber view during a short end-expiration pause. High frame rates (>150 frames/s) were obtained by reducing sector angles, and pulse repetition frequency was adjusted visually to avoid aliasing. ${ }^{17}$ All measurements were made in triplicate following international guidelines. ${ }^{18}$

Each multipoint of the PAMP-CO relationship was fitted to the following Linehan's distensibility model, ${ }^{1}$ allowing the calculation of a distensibility $\alpha$ index expressed in \% change in resistive vessel diameter per $\mathrm{mmHg}$ increase in transmural pressure during exercise by the equation PAMP = $\left[\left((1+\alpha \mathrm{LAP})^{5}+5 \alpha \cdot \mathrm{R}_{0} \cdot \mathrm{CO}\right)^{1 / 5}-1\right] / \alpha$, where $\mathrm{R}_{0}$ is total PVR (TPR) at rest and LAP, PAMP, and CO are measured at identical exercise level.

\section{Cardio-pulmonary exercise testing}

The CPET were performed as previously reported ${ }^{14}$ with breath by breath measurements of $\mathrm{VO}_{2}, \mathrm{VCO}_{2}$, and $\mathrm{VE}$ on a cycle ergometer at progressively increased workload (HypAir; Medisoft; Dinant, Belgium) of 10-30 W/min until volitional fatigue for an optimal test duration between 10 and 12 min for all subjects. $\mathrm{SpO}_{2}$ and $\mathrm{HR}$ were measured continuously. $\mathrm{BP}$ was measured every minute. The ventilatory threshold $\left(\mathrm{V}_{\mathrm{T}}\right)$ was measured by the $\mathrm{V}$-slope method on the $\mathrm{VCO}_{2}$ vs. $\mathrm{VO}_{2}$ relationship and $\mathrm{V}_{\mathrm{E}} \mathrm{NCO}_{2}$ ratio was then recorded. Oxygen pulse was calculated as $\mathrm{VO}_{2} / \mathrm{HR}$.

\section{Statistics}

Results are presented as mean \pm SD. The statistical analysis consisted of repeated measures analysis of variance calculations. When the $F$ ratio of 
the analysis of variance reached a $P<0.05$ critical value, Student's $t$-tests were applied for age group comparisons and exercise vs. rest comparisons. Correlations were calculated by Pearson's linear regression analysis. $P$ values $<0.05$ were considered significant.

\section{Results}

\section{Anthropomorphic data}

Height, weight, body mass index (BMI), and BSA regardless of sex increased during adolescence until the end of growth (Table 1). Height, weight, and BSA were higher in adult men compared to adult women with no sex-related differences in BMI (not shown).

\section{Haemodynamics}

As shown in Table 2, at rest there were no between-group differences for BP, CI, PAMP, and indexed TPR (TPRI), while SV was lower and $\mathrm{HR}$ higher in adolescents. At maximal exercise, adolescents showed identical $\mathrm{Cl}$ but lower BP, SV, CO compared to adults. Middle-aged adults had lower maximal HR and higher maximal SV, TPRI, and PAMP as compared to young adults.

The PAMP-Cl slope was lower in adolescents and increased with age (Figure 1). The CO-workload relationships were not different in the three age groups (all $0.06 \pm 0.01 \mathrm{~L} / \mathrm{min} / \mathrm{W}$ ). The PAMP-CO slopes were increased in the middle-aged adults compared to the two other age groups (Table 2) and so were the PASP-W slopes which were of $0.13 \pm 0.04,0.13 \pm 0.03$, and $0.22 \pm 0.06 \mathrm{mmHg} / \mathrm{W}$, respectively $(P<0.001$ compared to adolescent and young adult groups. The pulmonary vascular distensibility factor $\alpha$ was higher in adolescents and decreased with age (Figure 2). The limits of normal of alpha estimated as mean - 2SD to mean + 2SD would be 1.0-2.2 in adolescents, $0.8-2.0$ in young adults and $0.5-1.9 \% / \mathrm{mmHg}$ in middleaged adults (Figure 2). On average, $\alpha$ dropped by $25 \%$ over 26 years' time from adolescence to middle-aged adulthood.

Exercise induced a significantly increase in all haemodynamic variables except for a decrease in TPRI and $\alpha$.

As shown in the Supplementary data online, Table S1, there were no sex-related differences in the three age groups at rest. At exercise higher maximal SV, $\mathrm{CO}$, and $\mathrm{Cl}$ were observed in men compared to women in all age groups. In adult men $\alpha$ tended to be lower than in women, but the difference did not reach significance.

\section{Right ventricular structure and function}

For the sake of clarity, because the exercise capacity varied among the subjects, only resting and maximum exercise RV function measurements are reported here. As shown in Table 3, exercise was associated with an increase in TAPSE and S', and a decrease in TAPSE/ PASP and S'/PASP. The changes in S' were of $12 \pm 5,13 \pm 4$, and $8 \pm 3$, respectively ( $P<0.001$ compared with the two younger age groups). The changes in PASP were of $20 \pm 6,23 \pm 5$, and $33 \pm 13$, respectively ( $P<0.001$ compared to the two younger age groups). The TAPSE/ PASP and S'/PASP were lower at rest and at exercise in the middleaged adults. The effects of age on TAPSE/PASP at rest and during exercise are shown in Figures 3 and 4, respectively. The limits of normal of TAPSE/PASP in adolescents, young adults, and middle age adults would be $0.9-1.6,0.9-1.6$, and $0.7-1.4 \mathrm{~mm} / \mathrm{mmHg}$, respectively at rest, and $0.4-1.3,0.5-1.1$, and $0.4-1.0 \mathrm{~mm} / \mathrm{mmHg}$, respectively at maximal exercise. The effects of age on S'/PASP at rest and during exercise are shown in Figures 5 and 6, respectively. The limits of normal of S'PASP in adolescents, young adults, and middle age adults would be $0.4-0.9,0.4-0.8$, and $0.4-0.8$, respectively at rest, and $0.3-0.8,0.4$ 0.7 , and $0.3-0.6$, respectively at exercise.

\section{Cardio-pulmonary exercise testing}

As shown in Table 4, workload (W), RER, $\mathrm{O}_{2}$ pulse, and absolute $\mathrm{VO}_{2}$ at maximal exercise were lower, while $\mathrm{V}_{\mathrm{E}} / \mathrm{VCO}_{2}$ was increased in the adolescents. $\mathrm{VO}_{2}$ max corrected for body weight and VT were not different between the age groups. As shown in the Supplementary data online, Table S2, $\mathrm{VO}_{2}$ max, $\mathrm{O}_{2}$ pulse, workload, were higher in men independently of age. $\mathrm{V}_{\mathrm{E}} / \mathrm{VCO}_{2}$ was higher in young and middle-aged adult women.

\section{Discussion}

The present results show that healthy adolescents have a higher pulmonary vascular distensibility than young or middle-aged adults, and better coupling of the RV to the pulmonary circulation at rest and at exercise than middle-aged adults. Our observations offer insight into age-related limits of normal of these measurements from late childhood to early adult life.

Even though available validation against invasive measurements is only indirect, ${ }^{3,4}$ exercise stress echocardiography of the pulmonary circulation to generate PAMP-flow relationships and resistive vessel distensibility calculations has been shown to be feasible and has allowed to disclose age-, sex-, environmental-related, and early stage disease alterations. The distensibility factor $\alpha$ has been shown to be higher in pre-menopausal women and lower in aging adults, ${ }^{19}$ lower in young adult men of Sub-Saharan African ascendance ${ }^{20}$ lower with chronic but not acute hypoxic exposure ${ }^{2,21}$ or diesel exhaust exposure, ${ }^{22}$ and lower in adolescents born by in vitro fertilization. ${ }^{14}$ The $\alpha$ factor has been reported to be decreased in patients with borderline hypertension, ${ }^{23}$ early or latent pulmonary vascular disease, ${ }^{5,24}$ and heart failure. ${ }^{6,24}$ A decreased pulmonary vascular distensibility results in higher PA pressures during exercise, which may be associated with insufficient RV function adaptation, RV-PA uncoupling and decreased aerobic exercise capacity, in healthy subjects ${ }^{19,25}$ and more so in heart failure patients. ${ }^{6,24}$ In this study, the pulmonary circulation was more distensible in adolescents compared to adults. Even though this was associated with a better RV-PA coupling as assessed by TAPSE/ PASP and S'/PASP ratios, aerobic exercise measured by $\mathrm{VO}_{2}$ max corrected for body weight was not different. This is likely related to other growth and maturation factors with more impact than RV function adaptation to afterload. ${ }^{26}$

The RV basically adapts to afterload by an increased contractility to preserve its coupling to the pulmonary circulation and flow output adapted to metabolic demand. ${ }^{7,8}$ The matching of RV function to afterload in exercising healthy subjects, has been demonstrated to be well preserved by invasive measurements of Ees and Ea (Ees/Ea). ${ }^{27}$ The TAPSE/PASP was recently shown to be superior to other echocardiographic estimates of the Ees/Ea ratio for the quantification of RV-PA coupling. ${ }^{10,28}$ In those studies, the tricuspid annular systolic velocity S' was not assessed. In this study, TAPSE and S' increased during exercise in all age groups, in keeping with previously 
Table 2 Haemodynamics at rest and at maximum exercise in adolescents and in young and middle-aged adults

\begin{tabular}{|c|c|c|c|c|c|c|}
\hline Variables & & Adolescents & Young adults & Middle-age adults & $\boldsymbol{F}$ & $P$ \\
\hline \multirow[t]{2}{*}{$\mathrm{mBP}(\mathrm{mmHg})$} & Rest & $82 \pm 9$ & $83 \pm 9$ & $86 \pm 10$ & 1.5 & 0.23 \\
\hline & Max & $102 \pm 14$ & $111 \pm 17^{* *}$ & $112 \pm 13^{* *}$ & 5.3 & 0.0062 \\
\hline \multirow[t]{2}{*}{$\mathrm{HR}$ (bpm) } & Rest & $91 \pm 13$ & $84 \pm 12^{* *}$ & $82 \pm 15^{* *}$ & 4.9 & 0.0096 \\
\hline & Max & $194 \pm 12$ & $190 \pm 11$ & $174 \pm 12 * * *, \$ \$$ & 32.0 & 0.00001 \\
\hline \multirow[t]{2}{*}{$\mathrm{SV}(\mathrm{mL})$} & Rest & $55 \pm 10$ & $66 \pm 17^{* *}$ & $64 \pm 18 *$ & 5.9 & 0.003 \\
\hline & Max & $71 \pm 19$ & $85 \pm 16^{* *}$ & $97 \pm 25 * * * \$$ & 12.7 & 0.00001 \\
\hline \multirow[t]{2}{*}{$\mathrm{CO}(\mathrm{L} / \mathrm{min})$} & Rest & $4.9 \pm 0.8$ & $5.4 \pm 1.3$ & $5.1 \pm 1.2$ & 2.3 & 0.1 \\
\hline & Max & $13.7 \pm 3.2$ & $16.1 \pm 3.2^{* *}$ & $16.6 \pm 5.0 * *$ & 6.3 & 0.002 \\
\hline \multirow[t]{2}{*}{$\mathrm{Cl}\left(\mathrm{L} / \mathrm{min} / \mathrm{m}^{2}\right)$} & Rest & $2.7 \pm 0.4$ & $2.6 \pm 0.8$ & $2.7 \pm 0.8$ & 0.01 & 0.9 \\
\hline & Max & $7.4 \pm 1.6$ & $7.7 \pm 1.5$ & $7.7 \pm 2.2$ & 0.4 & 0.64 \\
\hline \multirow[t]{2}{*}{ PAMP $(\mathrm{mmHg})$} & Rest & $16.1 \pm 1.6$ & $16.0 \pm 1.6$ & $16.8 \pm 2.6$ & 1.4 & 0.25 \\
\hline & Max & $28.2 \pm 4.1$ & $29.8 \pm 3.5$ & $37.3 \pm 8.7 * * *, \$ \$ \$$ & 25.9 & 0.00001 \\
\hline \multirow[t]{2}{*}{ TPRI (Wood units $/ \mathrm{m}^{2}$ ) } & Rest & $6.2 \pm 1.1$ & $6.4 \pm 1.4$ & $6.7 \pm 2.0$ & 1.4 & 0.26 \\
\hline & Max & $3.8 \pm 0.7$ & $4.0 \pm 0.7$ & $5.0 \pm 0.9 * * * \$ \$ \$$ & 24.6 & 0.00001 \\
\hline PAMP-CO slope $(\mathrm{mmHg} / \mathrm{L} / \mathrm{min})$ & Total & $1.4 \pm 0.4$ & $1.4 \pm 0.4$ & $1.6 \pm 0.4^{*} \$ \$ \$$ & 6.2 & 0.003 \\
\hline PAMP-Cl slope $\left(\mathrm{mmHg} / \mathrm{L} / \mathrm{min} / \mathrm{m}^{2}\right)$ & & $2.5 \pm 0.7$ & $2.8 \pm 0.8^{*}$ & $3.4 \pm 0.8 * * * \$ \$$ & 13.6 & 0.00001 \\
\hline$\alpha(\% / \mathrm{mmHg})$ & & $1.60 \pm 0.31$ & $1.39 \pm 0.29 * *$ & $1.20 \pm 0.35 * * * \$$ & 15.3 & 0.00001 \\
\hline
\end{tabular}

All variables were significantly increased or decreased at maximum exercise.

$\mathrm{Cl}$, cardiac index; CO, cardiac output; HR, heart rate; mBP, mean blood pressure; PAMP, mean pulmonary arterial pressure; SV, stroke volume; TPRI, indexed total pulmonary vascular resistance.

$* P<0.05$ vs. adolescents.

$* * P<0.01$ vs. adolescents.

$* * * P<0.001$ vs. adolescents.

$\$ p<0.05$ young vs. middle-age adults.

$\$ \$ P<0.01$ young vs. middle-age adults.

$\$ \$ P<0.001$ young vs. middle-age adults.

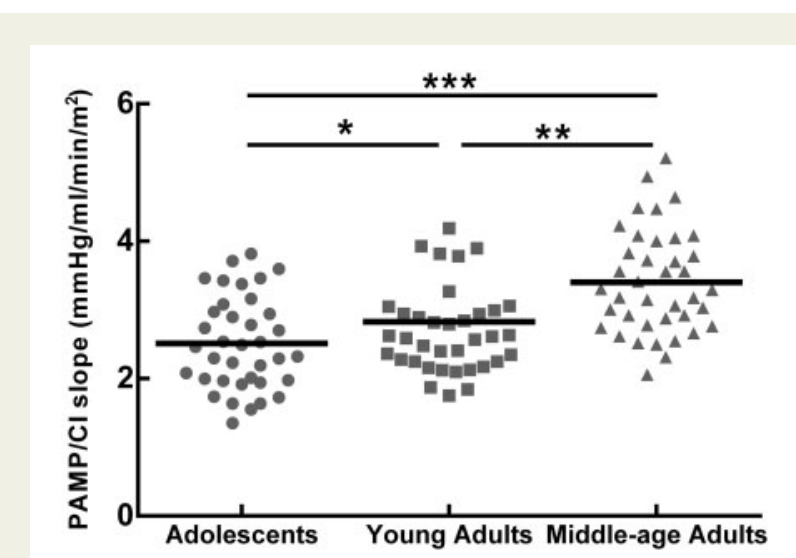

Figure I Influence of age on PAMP-Cl slope. PAMP-Cl slopes in adolescents (circles), young adults (squares), and middle-aged adults (triangles). Horizontal bars indicate the group means. The slope of the pressure-flow relationship increased with aging. $* P<0.05$, $* * P$ $<0.01$, and $* * * P<0.001$.

demonstrated increase in contractility to preserve RV-PA coupling. ${ }^{27}$ Yet the ratios of TAPSE or S' to PASP decreased with exercise, though less so in adolescents. Why the TAPSE/PASP or the S'/PASP decrease during exercise while the Ees/Ea does not is unclear. We may speculate that during exercise, as opposed to resting condition,

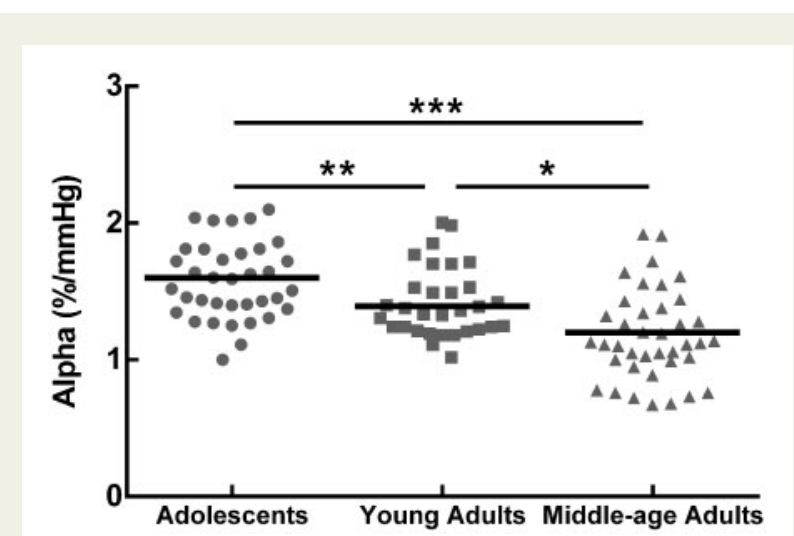

Figure 2 Influence of age on distensibility factor $\alpha$. Pulmonary vascular distensibility factor $\alpha$ in adolescents (circles), young adults (squares), and middle-aged adults (triangles). Horizontal bars indicate the means. Pulmonary vascular distensibility decreased with aging. $* P<0.05, * * P<0.01$, and $* * * P<0.001$.
RV systolic function might rely more on transverse rather than longitudinal shortening. It also may be that TAPSE as a measure of Ees and PASP as a measure of Ea lack specificity and proportional change in numbers compared to those of elastance measurements. This study nevertheless suggests that RV-PA coupling is better maintained in 


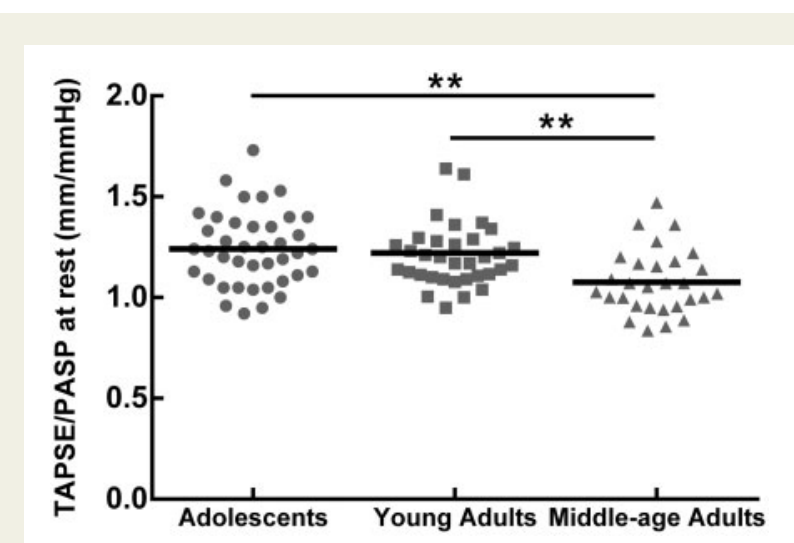

Figure 3 Age-related TAPSE/PASP at rest. TAPSE to PASP ratio at rest in adolescents (circles), young adults (squares), and middleaged adults (triangles). Resting TAPSE/PASP, a surrogate for RV-PA coupling, decreased in middle-aged adults. Horizontal bars indicate the means. $* * P<0.01$

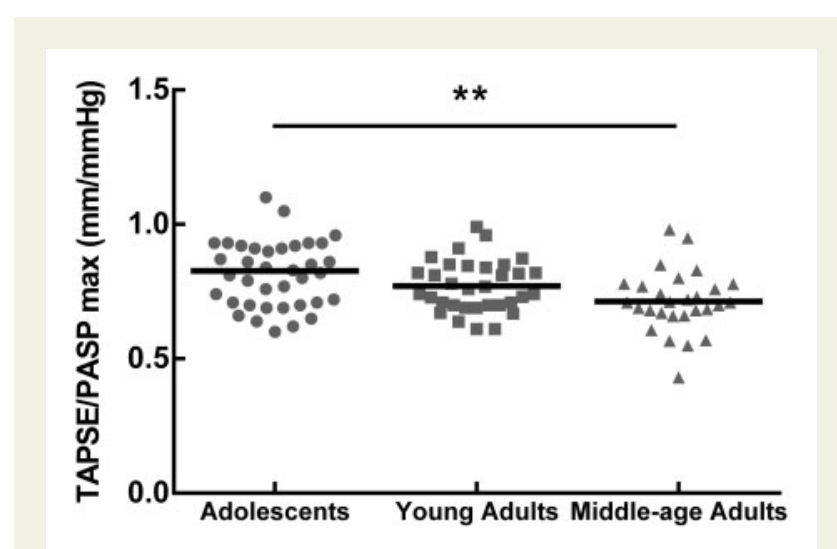

Figure 4 Age-related TAPSE/PASP at maximum exercise. TAPSE to PASP ratio at maximum exercise in adolescents (circles), young adults (squares), and middle-aged adults (triangles). Horizontal bars indicate the means. Maximal exercise TAPSE/PASP decreased in middle-aged adults. $* * p<0.01$.

adolescents, possibly in relation to more distensible pulmonary circulation.

A previous large-scale study on 1168 healthy subjects aged from 16 to 93 years showed that the TAPSE/PASP decreases with aging, but significantly so only after 60 years. ${ }^{12}$ In that study, the TAPSE/ PASP was on average higher in men compared to women on average by $0.2 \mathrm{~mm} / \mathrm{mmHg}$. In this study, there were no significant male vs. female differences in the TAPSE/PASP, which was, however, decreased in the middle-aged adults. In a smaller size study on 90 healthy adults aged from 19 to 63 years, the TAPSE/PASP decreased with aging following the equation TAPSE/PASP $=-0.013 \times$ age $+1.83 .{ }^{13}$ While in this study, TAPSE/PASP was similar to previously reported, the prediction equation over-estimated the measurements in adolescents, young, and middle-age adults by an average of $0.75,0.38$, and $0.23 \mathrm{~mm} / \mathrm{mmHg}$, respectively.

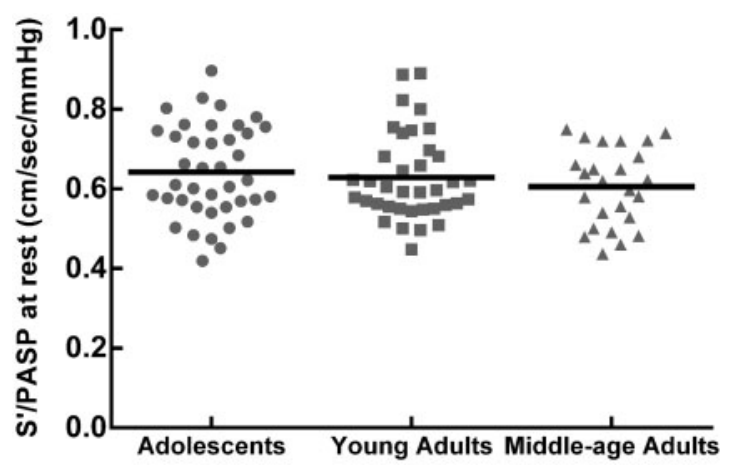

Figure 5 Age-related S'/PASP at rest. Tricuspid annular systolic velocity (S') to PASP ratio, a surrogate for RV-PA coupling, at rest in adolescents (circles), young adults (squares), and middle-aged adults (triangles). Aging did not affect the S'/PASP ratio.

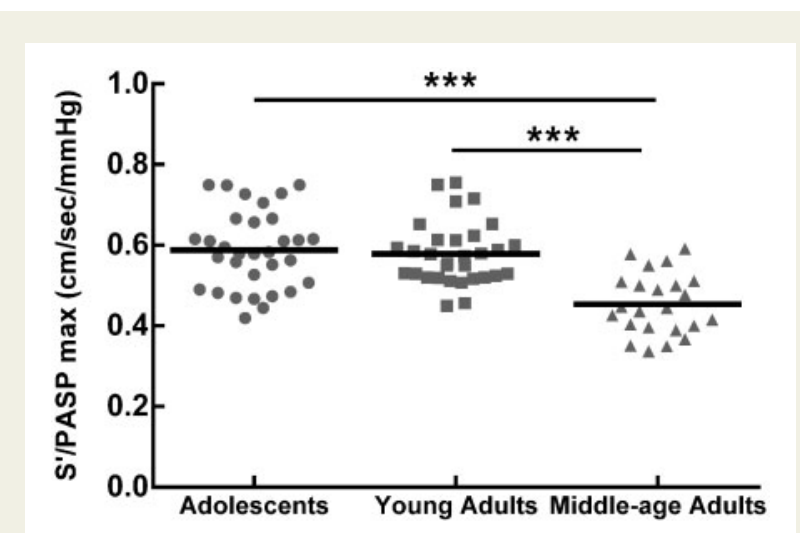

Figure 6 Age-related S'/PASP at maximum exercise. Tricuspid annular systolic velocity (S') to PASP ratio at maximum exercise in adolescents (circles), young adults (squares), and middle-age adults (triangles). S'/PASP decreased at exercise in middle-aged adults. Horizontal bars indicate the means. $* * * P<0.001$.

This study did not evaluate other indices of RV-PA coupling derived from 2D measurements as previously reported. ${ }^{29,30}$ Several such indices have been shown to be inferior to the TAPSE/PASP in predicting Ees/Ea. ${ }^{10}$ Exercise-induced changes in $S^{\prime}$ in the present study were quantitatively the same as exercise-induced changes in TAPSE, suggesting that the S'PASP could be used as alternative or an internal control to assess RV-PA coupling. However, the S'/PASP in the present study was not affected by aging at rest, which indirectly suggests inferior sensibility compared to TAPSE/PASP.

The present results confirm that aerobic exercise capacity increases gradually with age during childhood and adolescence. The kinetics of this evolution are different in girls and boys related to pubertal hormonal changes reaching a peak in $\mathrm{VO}_{2}$ max earlier in girls compared to boys. However, $\mathrm{VO}_{2} \mathrm{max}$ in children is not agedependent when corrected by body weight. ${ }^{26}$ On the other hand, the maximal workload, endurance time, maximum average running 
Table 3 Right ventricular function at rest and at maximum exercise in adolescents and in young and middle-age adults

\begin{tabular}{|c|c|c|c|c|c|c|}
\hline Variables & & Adolescents & Young adults & Middle-age adults & $\boldsymbol{F}$ & $P$ \\
\hline \multirow[t]{2}{*}{ TAPSE (mm) } & Rest & $24 \pm 3$ & $25 \pm 3$ & $24 \pm 3$ & 3.0 & 0.07 \\
\hline & Max & $33 \pm 4$ & $34 \pm 3$ & $33 \pm 4$ & 1.4 & 0.26 \\
\hline \multirow[t]{2}{*}{ TAPSE/PASP $(\mathrm{mm} / \mathrm{mmH})$} & Rest & $1.24 \pm 0.18$ & $1.22 \pm 0.17$ & $1.07 \pm 0.18 * *, \$ \$$ & 5.2 & 0.008 \\
\hline & $\operatorname{Max}$ & $0.86 \pm 0.24$ & $0.80 \pm 0.15$ & $0.72 \pm 0.15^{* *}$ & 3.7 & 0.04 \\
\hline \multirow[t]{2}{*}{$\mathrm{S}^{\prime}(\mathrm{cm} / \mathrm{s})$} & Rest & $15 \pm 2$ & $14 \pm 2$ & $13 \pm 2$ & 2.5 & 0.08 \\
\hline & $\operatorname{Max}$ & $26 \pm 5$ & $27 \pm 4$ & $21 \pm 3 * * * \$ \$ \$$ & 11.1 & 0.0001 \\
\hline \multirow[t]{2}{*}{$\mathrm{S}^{\prime} / \mathrm{PASP}(\mathrm{cm} / \mathrm{s} / \mathrm{mmHg})$} & Rest & $0.64 \pm 0.12$ & $0.63 \pm 0.11$ & $0.62 \pm 0.09$ & 0.3 & 0.74 \\
\hline & $\operatorname{Max}$ & $0.58 \pm 0.12$ & $0.58 \pm 0.08$ & $0.45 \pm 0.08 * * * \$ \$ \$$ & 10.9 & 0.0001 \\
\hline
\end{tabular}

All variables were significantly increased or decreased at maximum exercise.

PASP, systolic pulmonary arterial pressure; S', tricuspid annular systolic velocity; TAPSE, tricuspid annular plane systolic excursion.

$* * P<0.01$ vs. adolescents.

$* * * P<0.001$ vs. adolescents.

${ }^{\$} P<0.01$ young vs. middle-age adults.

$\$ \$ P<0.001$ young vs. middle-age adults.

Table 4 Cardio-pulmonary exercise testing

\begin{tabular}{|c|c|c|c|c|c|}
\hline Variables & Adolescents & Young adults & Middle-age adults & $\boldsymbol{F}$ & $P$ \\
\hline $\mathrm{VO}_{2} \max (\mathrm{L} / \mathrm{min})$ & $2.3 \pm 0.4$ & $2.8 \pm 0.7 * *$ & $3.0 \pm 0.9 * * *$ & 10.2 & 0.00001 \\
\hline $\mathrm{VO}_{2} \max (\mathrm{mL} / \mathrm{kg} / \mathrm{min})$ & $45 \pm 8$ & $43 \pm 7$ & $44 \pm 11$ & 0.2 & 0.8 \\
\hline Workload max (W) & $163 \pm 31$ & $206 \pm 53 * *$ & $260 \pm 100 * * * \$ \$$ & 20.6 & 0.00001 \\
\hline RER max & $1.14 \pm 0.07$ & $1.18 \pm 0.09 *$ & $1.21 \pm 0.10^{* *}$ & 5.3 & 0.006 \\
\hline $\mathrm{O}_{2}$ pulse $\max (\mathrm{mL} /$ beat $)$ & $11.9 \pm 2.7$ & $14.7 \pm 3.4 * *$ & $18.7 \pm 5.8^{* * * * \$ \$}$ & 24.0 & 0.006 \\
\hline \multicolumn{6}{|c|}{ Ventilatory threshold } \\
\hline $\mathrm{VO}_{2}\left(\% \mathrm{VO}_{2} \max \right)$ & $66 \pm 8$ & $64 \pm 8$ & $64 \pm 9$ & 0.7 & 0.5 \\
\hline $\mathrm{V}_{\mathrm{E}} \mathrm{NCO}_{2}$ & $31 \pm 3$ & $30 \pm 3^{*}$ & $29 \pm 3^{*}$ & 3.2 & 0.04 \\
\hline
\end{tabular}

RER, respiratory exchange ratio; $\mathrm{VCO}_{2}$, carbon dioxide output; $\mathrm{V}_{\mathrm{E}}$, ventilation; $\mathrm{VO}_{2}$ max, maximum oxygen uptake.

$* P<0.05$ vs. adolescents.

$* * P<0.01$ vs. adolescents.

$* * * P<0.001$ vs. adolescents.

${ }^{\$ \$} P<0.01$ young vs. middle-age adults.

${ }^{\$ \$} P<0.001$ young vs. middle-age adults.

speed increase continuously with age in relation to increased body dimensions and mechanical efficiency of muscle work. ${ }^{31}$ Higher $\mathrm{V}_{\mathrm{E}} / \mathrm{VCO}_{2}$ has also been previously reported in children, ${ }^{26}$ and in premenopausal women vs. adult men. ${ }^{31}$ These differences are explained by higher chemosensitivity in children and in adult women, the latter being related to progesterone secretion.

A limitation of this study is in the exclusive use of echocardiographic measurements without validation against other imaging modalities or invasive measurements. Non-invasive exercise stress testing of the pulmonary circulation using Doppler echocardiography has been previously indirectly validated by good agreement with invasive measurements in healthy subjects ${ }^{3,4,29}$ and in $\mathrm{PH}$ patient, ${ }^{32}$ but this has been rigorously demonstrated for all the components of the PVR equation only in resting conditions. ${ }^{33}$ Echocardiographic estimates of PAP from the maximum velocity of tricuspid regurgitation during exercise have recently been shown to be associated with only minimal bias at Bland and Altman analysis, demonstrating acceptable accuracy, but limits of agreement were wide indicating limited precision. ${ }^{34}$ Nineteen percent of the subjects were excluded at preliminary screening for insufficient quality of tricuspid regurgitation signals to estimate PAP. This is higher than $16 \%$ reported in previous studies in our laboratory. ${ }^{19}$ Even higher rates of insufficient quality exercise echocardiographies in healthy volunteers have been reported by others, ${ }^{34}$ showing that the estimation of PAP from tricuspid regurgitant velocities requires expertise. Whether the excluded subjects might have influenced the reported results is not known. The TAPSE/ PASP ratio has been validated by uni- and multivariable analysis of predictors of Ees/Ea among usual echocardiographic assessments of the RV. ${ }^{10}$ In general imaging estimates of the PVR equation or RV-PA coupling, though accurate, may suffer from insufficient precision to allow for individual clinical decisions.

This study did not include children aged $<12$ years, which is another limitation to the definition of still needed exhaustive paediatric limits of normal for the pulmonary circulation and RV function. 
However, this would require another study with adapted technology and study design.

A final limitation is that CPET and exercise stress echocardiography were performed on different days, which could have increased the variability in the measurements. However, simultaneous echocardiography and CPET is technically very difficult because of multiplicity of operators, connections, and measurements which could be another potential cause of variability in the results.

In conclusion, adolescents compared to young or middle-aged adults have a more distensible pulmonary circulation and better indices of RV-PA coupling but of insufficient magnitude to allow for body-size-corrected aerobic exercise capacity.

\section{Supplementary data}

Supplementary data are available at European Heart Journal - Cardiovascular Imaging online.

\section{Funding}

S.C. was supported by an European Respiratory Society (ERS) Pulmonary Arterial Hypertension short term research training fellowship (20145264). Y.M. was supported by a grant from the Cardiac Surgery Funds, Belgium.

\section{Conflict of interest: none declared.}

\section{References}

1. Linehan JH, Haworth ST, Nelin LD, Krenz GS, Dawson CA. A simple distensible model for interpreting pulmonary vascular pressure-flow curves. J Appl Physiol 1992;73:987-94

2. Reeves JT, Linehan JH, Stenmark KR. Distensibility of the normal human lung circulation during exercise. Am J Physiol Lung Cell Mol Physiol 2005;288:419-25.

3. Lewis GD, Bossone E, Naeije R, Grünig E, Saggar R, Lancellotti $P$ et al. Pulmonary vascular hemodynamic response to exercise in cardiopulmonary diseases. Circulation 2013;128:1470-9.

4. Naeije R, Vanderpool R, Dhakal BP, Saggar R, Saggar R, Vachiery JL et al. Exercise-induced pulmonary hypertension physiological basis and methodological concerns. Am J Respir Crit Care Med 2013;187:576-83.

5. Lau EM, Chemla D, Godinas L, Zhu K, Sitbon O, Savale L et al. Loss of vascular distensibility during exercise is an early hemodynamic marker of pulmonary vascular disease. Chest 2016;149:353-61.

6. Malhotra R, Dhakal BP, Eisman AS, Pappagianopoulos PP, Dress A, Weiner RB et al. Pulmonary vascular distensibility predicts pulmonary hypertension severity, exercise capacity, and survival in heart failure. Circ Heart Fail 2016;9:1-11.

7. Vonk Noordegraaf A, Chin KM, Haddad F, Hassoun PM, Hemnes AR, Hopkins SR et al. Pathophysiology of the right ventricle and of the pulmonary circulation in pulmonary hypertension: an update. Eur Respir J 2019;53:1801900.

8. Sanz J, Sánchez-Quintana D, Bossone E, Bogaard HJ, Naeije R. Anatomy, function, and dysfunction of the right ventricle: JACC state-of-the-art review. J Am Coll Cardiol 2019;73:1463-82.

9. Rudski LG, Gargani L, Armstrong WF, Lancellotti P, Lester SJ, Grünig E et al. Stressing the cardiopulmonary vascular system: the role of echocardiography. J Am Soc Echocardiogr 2018;31:527-50.

10. Tello K, Wan J, Dalmer A, Vanderpool R, Ghofrani HA, Naeije R et al. Validation of the tricuspid annular plane systolic excursion/systolic pulmonary artery pressure ratio for the assessment of right ventricular-arterial coupling in severe pulmonary hypertension. Circ Cardiovasc Imaging 2019;12:e009047.

11. Guazzi M. Use of TAPSE/PASP ratio in pulmonary arterial hypertension: an easy short-cut in a congested road. Int J Cardiol 2018;266:242-4.

12. Ferrara F, Rudski LG, Vriz O, Gargani L, Afilalo J, D’Andrea A et al. Physiologic correlates of tricuspid annular plane systolic excursion in 1168 healthy subjects. Int J Cardiol 2016;223:736-43.

13. D'Alto M, Pavelescu A, Argiento P, Romeo E, Correra A, Di Marco GM et al. Echocardiographic assessment of right ventricular contractile reserve in healthy subjects. Echocardiography 2017;34:61-8.
14. Forton K, Motoji Y, Pezzuto B, Caravita S, Delbaere A, Naeije R et al. Decreased pulmonary vascular distensibility in adolescents conceived by in vitro fertilization. Hum Reprod 2019;34:1799-808.

15. Forton K, Motoji Y, Deboeck G, Faoro V, Naeije R. Effects of body position on exercise capacity and pulmonary vascular pressure-flow relationships. J Appl Physiol 2016;121:1145-50.

16. Chemla D, Castelain V, Humbert M, Hebert JL, Simonneau G, Lecarpentier $Y$ et al. New formula for predicting mean pulmonary artery pressure using systolic pulmonary artery pressure. Chest 2004;126:1313-7.

17. Daubeney PEF, Blackstone E, Weintraub R, Slavik Z, Scanlon J, Webber S Relationship of the dimension of cardiac structures to body size: an echocardiographic study in normal infants and children. Cardiol Young 1999;9:402-10.

18. Rudski LG, Lai WW, Afilalo J, Hua L, Handschumacher MD, Chandrasekaran K et al. Guidelines for the echocardiographic assessment of the right heart in adults: a report from the American Society of Echocardiography endorsed by the European Association of Echocardiography, a registered branch of the European Society of Cardiology, and the Canadian Society of Echocardiography. J Am Soc Echocardiogr 2010;23:685-713.

19. Argiento P, Vanderpool RR, Mulè M, Russo MG, D'Alto M, Bossone E et al. Exercise stress echocardiography of the pulmonary circulation: limits of normal and sex differences. Chest 2012;142:1158-65.

20. Simaga B, Vicenzi M, Faoro V, Caravita S, Di Marco G, Forton K et al. Pulmonary vascular function and exercise capacity in black sub-Saharan Africans. J Appl Physiol 2015;119:502-7.

21. Groepenhoff $H$, Overbeek MJ, Mulè $M$, van der Plas $M$, Argiento P, Villafuerte FC et al. Exercise pathophysiology in patients with chronic mountain sickness. Chest 2012;142:877-84.

22. Wauters A, Vicenzi M, De Becker B, Riga JP, Esmaeilzadeh F, Faoro V et al. At high cardiac output, diesel exhaust exposure increases pulmonary vascular resistance and decreases distensibility of pulmonary resistive vessels. Am J Physiol Heart Circ Physiol 2015;309:2137-44.

23. Vriz O, Argiento P, D'Alto M, Ferrara F, Vanderpool R, Naeije R et al. Increased pulmonary vascular resistance in early stage systemic hypertension: a resting and exercise stress echocardiography study. Can J Cardiol 2015;31: 537-43.

24. Singh I, Oliveira RKF, Naeije R, Rahaghi FN, Oldham WM, Systrom DM et al. Pulmonary vascular distensibility and early pulmonary vascular remodeling in pulmonary hypertension. Chest 2019;156:724-32.

25. Lalande S, Yerly P, Faoro V, Naeije R. Pulmonary vascular distensibility predicts aerobic capacity in healthy individuals. J Physiol 2012;590:4279-88.

26. Ten Harkel AD, Takken T, Van Osch-Gevers M, Helbing W. Normal values for cardiopulmonary exercise testing in children. Eur J Cardiovas Prev Rehabil 2011;18: 48-54.

27. Spruijt OA, de Man FS, Groepenhoff H, Oosterveer F, Westerhof N, Vonk Noordegraaf $A$ et al. The effects of exercise on right ventricular contractility and right ventricular-arterial coupling in pulmonary hypertension. Am J Respir Crit Care Med 2015;191:1050-7.

28. Richter MJ, Peters D, Ghofrani HA, Naeije R, Roller F, Sommer N et al. Evaluation and prognostic relevance of right ventricular-arterial coupling in pulmonary hypertension. Am J Respir Crit Care Med 2020;201:116-9.

29. Claessen G, La Gerche A, Voigt JU, Dymarkowski S, Schnell F, Petit T et al. Accuracy of echocardiography to evaluate pulmonary vascular and RV function during exercise. JACC Cardiovasc Imaging 2016;9:532-43.

30. Pratali L, Allemann Y, Rimoldi SF, Faita F, Hutter D, Rexhaj E et al. RV contractility and exercise-induced pulmonary hypertension in chronic mountain sickness: a stress echocardiographic and tissue Doppler imaging study. JACC CardiovasC Imaging 2013;6:1287-97.

31. Cooper DM, Leu S-Y, Galassetti P, Radom-Aizik S. Dynamic interactions of gas exchange, body mass, and progressive exercise in children. Med Sci Sports Exerc 2014;46:877-86.

32. Caravita S, Yerly P, Baratto C, Dewachter C, Faini A, Rimouche A et al. Non-invasive versus invasive pressure/flow relationship of the pulmonary circulation: bias and error. Eur Respir J 2019;54:1900881.

33. D'Alto M, Romeo E, Argiento P, D'Andrea A, Vanderpool R, Correra A et al. Accuracy and precision of echocardiography versus right heart catheterization for the assessment of pulmonary hypertension. Int J Cardiol 2013; 168:4058-62.

34. van Riel AC, Opotowsky AR, Santos M, Rivero JM, Dhimitri A, Mulder BJ et al. Accuracy of echocardiography to estimate pulmonary artery pressures with exercise: a simultaneous invasive-noninvasive comparison. Circ Cardiovasc Imaging 2017; 10:e005711. 